\title{
The Effects of Changes in Hand Position on the Electromyographic Activities of the Shoulder Stabilizer Muscles during Push-up Plus Exercises on Unstable Surfaces
}

\author{
Sangyong Lee, PhD, PT ${ }^{1)}$, Daehee Lee, PhD, PT ${ }^{1)}$, Jungseo Park, Ms, PT ${ }^{1)}$ \\ 1) Department of Physical Therapy, Youngdong University: 12 Youngdong-eup, Youngdong-gun, \\ Chungbuk 370-701 Republic of Korea. TEL: +82 43-740-1404, FAX: +82 43-740-1109, E-mail: \\ emotion526@nate.com
}

\begin{abstract}
Purpose] The purpose of the present study was to examine the effects of changes in hand position on the electromyographic activities of the shoulder stabilizer muscles during push-up plus exercises (PUPE) on unstable surfaces. [Subjects] The subjects of the present study were normal adults in their 20s ( $n=15)$. PUPEs were performed with the hands in the neutral positions (NP), internal rotation positions (IRP), and external rotation positions (ERP) using a sling device for the unstable surface. [Methods] We measured the electromyographic activities of the wrist flexor (WF), the wrist extensor (WE), the biceps brachii (BB), the triceps brachii (TB), the upper trapezius (UT), the lower trapezius (LT), the serratus anterior (SA), and the pectoralis major (PM). The muscle activites were compared and analyzed using electromyography. [Results] When hand position changed, WF activity in NP, and SA activity in ERP, were significantly different from their respective activities in the other positions. [Conclusion] To selectively enhance the electromyographic activity of the SA during PUPE using a sling device as an unstable surface, we consider performance of PUPE in ERP is an effective intervention.

Key words: Push-up plus, Hand position, Sling device
\end{abstract}

(This article was submitted Sep. 14, 2012, and was accepted Oct. 9, 2012)

\section{INTRODUCTION}

The scapula stabilizer muscles provide stability to the shoulder joint and act together with the rotator cuff muscles in upward rotation of the shoulder bone. The scapula stabilizer muscles of the serratus anterior muscle (SA), the upper trapezius muscle (UT), and the lower trapezius muscle (LT) act in upward rotation of the scapula ${ }^{1)}$. SA is considered of primary importance for scapula stabilization, and plays the role of a lever in the protraction of the scapula toward the vertical axis of rotation of the sternoclavicular joint. It acts in abduction and upward rotation of the scapula toward the vertical axis of rotation, maintaining the scapula parallel to the ribs ${ }^{2}$. Abnormal electromyographic activity of the SA is associated with scapula instability. Weakening of the SA is found in baseball players with shoulder joint instability, workers with impingement syndrome of the shoulder joint, and swimmers with pain in the shoulder joint. Decreases in the EMG activity of SA are associated with abnormal scapula movements ${ }^{3-5}$.

Those whose functional scapula movements are suppressed due to unbalanced coupling of forces on the muscles can recover normal position of the shoulder bones through isometric exercises and training for stability. To this end, closed chain exercises are frequently used in upper extremity kinesiotherapy programs ${ }^{6}$. Closed chain exercises effective for scapula stabilization include push-up and push-up plus exercises (PUPE), and isometric ball exercises. The push-up exercise starts with the arm in the prone position. The elbow joint is bent and the body is lowered close to, but not touching the ground, and then the body is raised by straightening the arm. When performing shoulder joint stabilization exercises, using unstable surfaces that induce dynamic movement is more effective than static and continuous exercises at eliciting the exertion of muscle force. In the case of push-up exercises, unstable surfaces are more effective at stimulating the proprioceptive sense of the shoulder joint and increasing muscle activity. PUPE is an exercise that adds scapula protraction following maximum extension of the elbow joints during the last stage of extension in the push-up exercise. In particular, sling exercises are a dynamic exercise method that reinforces more muscles involved in stability than exercises on fixed surfaces. These exercises also improve the stability of the trunk and proximal joints.

The sling exercise uses a sling device to hang the exerciser from a swinging rope. This exercise strengthens the muscles and restores joint stability by stimulating the proprioceptive sense through its unstable nature. Moseley et al. ${ }^{7}$ recommended PUPE as an effective closed kinetic chain training method for training the muscles that cooperate in scapula stabilization and selectively reinforce SA. Although many 


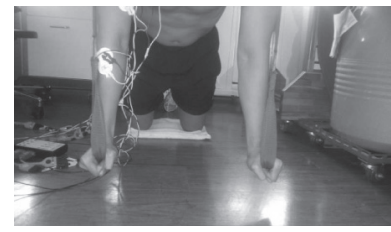

Fig. 1. Hand-position (neutral position) on the sling

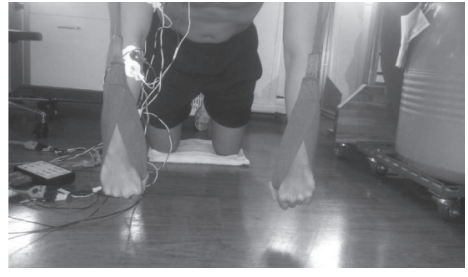

Fig. 2. Hand-position (internally rotated position) on the sling

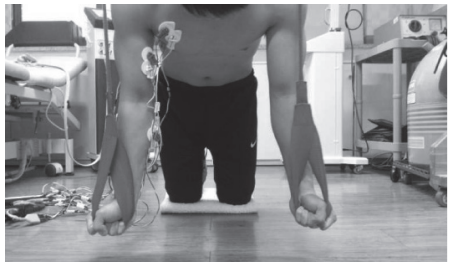

Fig. 3. Hand-position (externally rotated position) on the sling studies have examined PUPE on stable surfaces, studies of the changes in shoulder stabilizer muscle activities in relation to hand position on unstable surfaces are still lacking.

Therefore, the present study examined the changes in the electromyographic activities of the shoulder stabilizer muscles with hand position during PUPE performed using a sling device as an unstable surface. PUPE is used as a closed chain exercise method in upper extremity exercise programs.

\section{SUBJECTS AND METHODS}

The subjects of the present study were 15 healthy young men in their 20s who were attending $\mathrm{Y}$ University in Chungbuk. The dominant hand of all subjects was the right hand. The subjects' mean age was $23.4 \pm 1.40$ years, their mean height was $174.27 \pm 4.91 \mathrm{~cm}$, and their mean weight was $72.07 \pm 9.53 \mathrm{~kg}$. All the study subjects voluntarily agreed to participate in the experiment after hearing explanations about the purpose of the present study and its exercise methods.

A sling device (Nordisk Terapi AS Kilsund Senteret, 4920 Stand) was used to provide the unstable surface while subjects performed PUPE. Each subject was instructed to adopt a four-foot crawling posture supporting the weight with both hands and knees placed at shoulder width apart with the two hands holding the sling device at a vertical height of $10 \mathrm{~cm}$ above the ground as the starting posture. In the four-foot crawling posture, the subjects were instructed to spread their arms and legs to shoulder width while maintaining the shoulder, hip and knee joints in $90^{\circ}$ flexion, as well as maintain a neutral position with the head, spine and pelvis in alignment in a straight line, and watch a point drawn on the floor. The elbow joints were completely extended and the ankle joints were in plantarflexion. To maintain the neutral position of the cervical region, the cervical region and the back were maintained in a straight line. The subject was instructed to perform a PUPE on the command, "start", and to maintain the PUPE position just above the ground for five seconds. PUPE was performed with the hands in the neutral position (NP) with the upper extremities in the neutral position, in the internal rotation position (IRP) with the upper extremities rotated $90^{\circ}$ internally, and in the external rotation position (ERP) with the upper extremities rotated $90^{\circ}$ externally were taken during the exercises (Figs. 1, 2, and 3). Data were discarded when the standardized starting posture, exercise and finishing postures were not correctly performed. A researcher measured the electromyo- graphic activities three times, repeatedly, after requesting the subject to adopt the correct positions. The subjects took a rest of two minutes after each session. The subjects were allowed to learn the movement from the four-foot crawling posture to the PUPE posture through practice for 10 minutes with oral instructions and demonstrations. At this time, the subjects were cautioned not to let their backs rise excessively in compensatory action. All experimental processes were conducted by one researcher who also attached the electrodes to minimize position variability between sublects. The hand positions were changed in a random order.

Changes in the electromyographic activities of the scapula stabilization-related muscles were measured using an MP150 (Biopac System, USA). To quantify the action potentials of all the muscles, maximum voluntary isometric contractions (MVIC) were performed in manual muscle testing positions. $\mathrm{Ag}-\mathrm{Ag} / \mathrm{Cl}$ (Biopac, diameter $2 \mathrm{~cm}$ ) electrodes were used for the measurement. Since the doninant hand of all the subjects was the right hand, surface electrodes were attached to the right upper extremities. The surface electrode for the wrist flexor (WF) was attached to the medial side at a point $1 / 3$ of the length of the forearm from the elbow joint, that for the wrist extensor (WE) was attached to the lateral side at a point $1 / 2$ of the length of the forearm from the elbow joint, that for the biceps brachii muscle (BB) was attached to the front side midway between the shoulder joint and the elbow joint, that for the triceps brachii muscle (TB) was attached to the back side midway between the shoulder joint and the elbow joint, that for the UT was attached midway between the spinous process and the acromion of $\mathrm{C} 7$, that for the LT was attached to the medial side at a point $1 / 4$ of the distance between the thoracic vertebra and the inferior angle of the scapula from the thoracic vertebra, that for the SA was attached to the anterior side of the latissimus dorsi muscle at the level of rib \#5 or 6, and that for the pectoralis major muscle (PM) was attached at a point $2 \mathrm{~cm}$ from the anterior axillary border of the medial side.

Electromyography signals were collected at a sampling rate of $1000 \mathrm{~Hz}$, full-wave rectified, band-pass filtered between $30-500 \mathrm{~Hz}$ using Acknowledge 3.81 (Biopac System, USA) software, and filtered at $60 \mathrm{~Hz}$ to remove noise. To standardize the data, the raw data were converted into Root Mean Square (RMS) values and the values measured during the middle three seconds of the measuring time of 5 seconds were collected after the first and last seconds of data. The resulting RMS values were divided by the maximum voluntary isometric contraction (MVIC) 
values to normalize the values as \%MVIC values.

The electromyographic activities of the muscles during PUPE in NP, IRP and ERP on an unstable surface were analyzed using repeated one-way ANOVA. A significance level, $\alpha$, of 0.05 was chosen and the data were statistically processed using the commercial statistical program SPSS version 12.0 for Windows.

\section{RESULTS}

In the present study, WF activity in NP, and SA activity in ERP, were significantly different $(p<0.05)$ from their respective activities in the other positions. However, WE, BB, TB, UT, LT and PM did not show any significant differences $(p>0.05)$ (Table 1).

\section{DISCUSSION}

In the present study, the electromyographic activities of WF, WE, BB, TB, UT, LT, SA and PM were examined while subjects performed PUPE, as closed chain exercises, as scapula stabilization exercises in NP, IRP and ERP using a sling device as an unstable surface.

Oh et al. ${ }^{8)}$ stated that, in contrast to exercises on stable surfaces, sling exercises using unstable surfaces were dynamic exercise methods that reinforce many muscles and improve the stability of proximal joints. Accordingly, in the present study, PUPEs were conducted using a sling device as an unstable surface and different hand positions.

A study by de Oliveira et al. ${ }^{9}$ reported finding no significant differences in SA between push up exercises on stable surfaces and the same exercises on unstable surfaces using gym balls. According to a study by Park and Yoo ${ }^{10)}$, PUPEs are more effective than push up exercises for the activation of SA. In particular, exercises on unstable surfaces are more effective than exercises on stable surfaces. However, a study by Lehmanetal ${ }^{11)}$ reported that when PUPEs were performed on stable and unstable surfaces, the electromyographic activity of SA showed significant differences between different heights of the feet regardless of the conditions of surfaces on which the hands were placed. Ludewig et al. ${ }^{6}$ reported that, while 30 healthy adults were performing push up exercises, the electromyographic activity of SA increased while that of the UT decreased. They concluded that push-up exercises were optimal for reinforcing SA. Choi ${ }^{12)}$ reported that, when patients with chronic shoulder joint pain that had persisted for at least three months performed push-up exercises for seven weeks, electromyographic activities of the anterior deltoid muscle and LT showed no significant differences, while that of UT significantly decreased and that of SA significantly increased.

Yoon et al. ${ }^{13)}$ reported that on stable surfaces, the electromyographic activity of SA was the highest in ERP and the lowest in NP. Lee et al. ${ }^{14)}$ reported that, when only NP and IRP were compared, higher electromyographic activity was shown in NP than in IRP. In the present study, the electromyographic activity of SA was the highest in ERP, similar to the results reported by Yoon et al. ${ }^{13)}$ This is because we used sling devices which are unstable surfaces. On unstable
Table 1. Changes in the electromyographic activities of the shoulder stabilizer muscles in relation to hand position

\begin{tabular}{cccc}
\hline Muscle & NP & IRP & ERP \\
\hline WF $^{*}$ & $20.7 \pm 16.2^{\mathrm{a}}$ & $18.2 \pm 13.2$ & $13.5 \pm 10.7$ \\
WE & $11.4 \pm 9.5$ & $12.3 \pm 17.7$ & $9.1 \pm 8.6$ \\
BB & $16.3 \pm 14.5$ & $14.9 \pm 18.4$ & $21.3 \pm 16.9$ \\
TB & $25.8 \pm 17.9$ & $25.1 \pm 14.9$ & $30.4 \pm 18.2$ \\
UT & $18.6 \pm 22.0$ & $13.6 \pm 10.5$ & $13.4 \pm 12.1$ \\
LT & $6.3 \pm 4.1$ & $8.5 \pm 8.4$ & $6.9 \pm 4.2$ \\
SA $^{* *}$ & $38.4 \pm 15.1$ & $34.3 \pm 15.2$ & $54.3 \pm 12.9$ \\
PM & $53.1 \pm 25.2$ & $41.3 \pm 18.1$ & $55.8 \pm 24.7$ \\
\hline
\end{tabular}

(unit:\%) Note. WF: wrist flexor, WE: wrist extensor, BB: biceps brachii, TB: triceps brachii, UT: upper trapezius, LT: lower trapezius, SA: serratus anterior, PM: pectoralis major, NP: neutral position, IRP: $90^{\circ}$ internal rotation position, ERP: $90^{\circ}$ external rotation position, ${ }^{\mathrm{a}}$ Mean $\pm \mathrm{SD},{ }^{* *}: \mathrm{p}<0.01,{ }^{*}: \mathrm{p}<0.05$

surface, BB and TB support the upper and lower side of the shoulder joints, while UT, LT and PM support the front and back sides to suppress movements of the shoulders, thereby providing stability. We consider that, on an unstable surface ERP allows greater stability than NP and IRP, and in consequence the electromyographic activity of SA was selectively increased.

Lee et al. ${ }^{14)}$ reported that electromyographic activity of the WF was higher in IRP than in NP. The results of the present study show that the electromyographic activity of WF was higher in NP than in ERP or IRP. Thus, the results of the two studies contradict each other. We consider that WF was activated more during PUPE on the sling, which is an unstable surface, in NP in order to maintain body stability.

The present study had some limitations. First, since the number of study subjects was small and all the subjects were normal adult males, the results cannot be generalized to all subjects. Second, since all the subjects had a dominant right hand only the muscles of the right extremity were measured; changes on the non-dominant side were not recorded. Third, the effects of changes in hand positions on the shoulder stabilizer muscles during PUPE on stable and unstable surfaces should be studied.

\section{REFERENCE}

1) Lear LJ, Gross MT: An electromyographical analysis of the scapular stabilizing synergists during a push-up progression. J Orthop Sports Phys Ther, 1998, 28: 146-157. [Medline]

2) Neumann DA: Kinesiology of the musculoskeletal system: foundation for physical rehabilitation. St. Louis: Mosby. 2002, pp 123-144.

3) Glousman R, Jobe F, Tibone J, et al.: Dynamic electromyographic analysis of the throwing shoulder with glenohumeral instability. J Bone Joint Surg Am, 1988, 70: 220-226. [Medline]

4) Ludewig PM, Cook TM: Alterations in shoulder kinematics and associated muscle activity in people with symptoms of shoulder impingement. Phys Ther, 2000, 80: 276-291. [Medline]

5) Scovazzo ML, Browne A, Pink M, et al.: The painful shoulder during freestyle swimming. An electromyographic cinematographic analysis of twelve muscles. Am J Sports Med, 1991, 19: 577-582. [Medline] [CrossRef]

6) Ludewig PM, Hoff MS, Osowski EE, et al.: Relative balance of serratus anterior and upper trapezius muscle activity during push-up exercises. Am J Sports Med, 2004, 32: 484-493. [Medline] [CrossRef]

7) Moseley JB Jr, Jobe FW, Pink M, et al.: EMG analysis of the scapular 
muscles during a shoulder rehabilitation program. Am J Sports Med, 1992, 20: 128-134. [Medline] [CrossRef]

8) Oh JS, Park JS, Kim SY: Comparison of muscle activity during a push-up on a suspension sling and fixed support. J Phys Ther Korea. 2003, 10: 29-40.

9) de Oliveira AS, de Morais Carvalho M, de Brum DP: Activation of the shoulder and arm muscles during axial load exercises on a stable base of support and on a medicine ball. J Electromyogr Kinesiol, 2008, 18: 472-479. [Medline] [CrossRef]

10) Park SY, Yoo WG: Differential activation of parts of the serratus anterio muscle during push-up variations on stable and unstable bases of support. J Electromyogr Kinesiol, 2011, 21: 861-867. [Medline] [CrossRef]
11) Lehman GJ, Gilas D, Patel U: An unstable support surface does not increase scapulothoracic stabilizing muscle activity during push up and push up plus exercises. Man Ther, 2008, 13: 500-506. [Medline] [CrossRef]

12) Choi JD: The effect of 7-week serratus anterior strengthening exercise on shoulder pain with serratus anterior weakness. Yonsei University. Dissertation of Doctorate Degree. 2008.

13) Yoon JY, Kim TH, Oh JS: Effect of hand positions in electromyographic Activity in scapulothoracic muscles during push-up plus. J Phys Ther Korea, 2010, 17: 8-15.

14) Lee SY, Jung JM, Hwangbo G: The effects on shoulder stabilizer activation of finger flexor activation during the push-up plus exercise. J Phys Ther Sci, 2011, 23: 575-577. [CrossRef] 